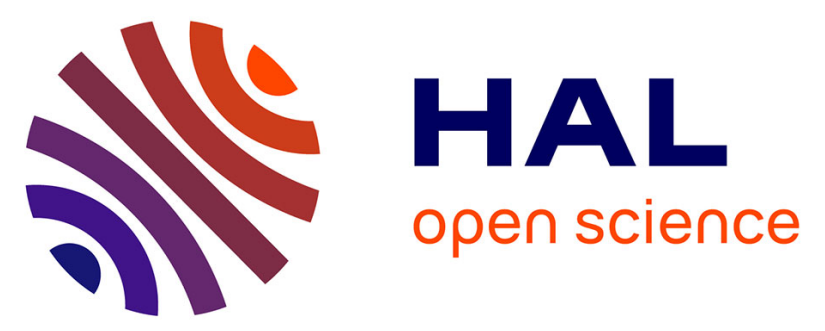

\title{
Weierstrass approach to asymptotic behavior characterization of critical imaginary roots for retarded differential equations
}

Alejandro Martínez-González, César Fernando Méndez Barrios, Silviu-Iulian

Niculescu, Jie Chen, Liliana Félix

\section{To cite this version:}

Alejandro Martínez-González, César Fernando Méndez Barrios, Silviu-Iulian Niculescu, Jie Chen, Liliana Félix. Weierstrass approach to asymptotic behavior characterization of critical imaginary roots for retarded differential equations. SIAM Journal on Control and Optimization, 2019, 57 (1), pp.1-22. 10.1137/17m1147135 . hal-01973973

\section{HAL Id: hal-01973973 \\ https://hal.science/hal-01973973}

Submitted on 8 Jan 2019

HAL is a multi-disciplinary open access archive for the deposit and dissemination of scientific research documents, whether they are published or not. The documents may come from teaching and research institutions in France or abroad, or from public or private research centers.
L'archive ouverte pluridisciplinaire HAL, est destinée au dépôt et à la diffusion de documents scientifiques de niveau recherche, publiés ou non, émanant des établissements d'enseignement et de recherche français ou étrangers, des laboratoires publics ou privés. 


\title{
WEIERSTRASS APPROACH TO ASYMPTOTIC BEHAVIOR CHARACTERIZATION OF CRITICAL IMAGINARY ROOTS FOR RETARDED DIFFERENTIAL EQUATIONS
}

\author{
A. MARTÍNEZ-GONZÁLEZ*, ${ }^{\dagger}$, C.-F. MÉNDEZ-BARRIOS*, S.-I. NICULESCU ${ }^{\dagger}, J^{*}$ CHEN ${ }^{\ddagger}$, \\ AND L. FÉLIX*
}

\begin{abstract}
This paper focuses on the analysis of the behavior of characteristic roots of timedelay systems, when the delay is subject to small parameter variations. The analysis is performed by means of the Weierstrass polynomial. More specifically, such a polynomial is employed to study the stability behavior of the characteristic roots with respect to small variations on the delay parameter. Analytic and splitting properties of the Puiseux series expansions of critical roots are characterized by allowing a full description of the cases that can be encountered. Several numerical examples encountered in the control literature are considered to illustrate the effectiveness of the proposed approach.
\end{abstract}

Key words. Weierstrass polynomial, Newton Diagram, Retarded Systems.

AMS subject classifications. $93 \mathrm{C} 02$

1. Introduction. There exist an abundant literature where the delay phenomenon has been associated with "undesired" behaviors or bad performance, (as, for examples, instabilities, oscillations, bandwidth sensitivity), as pointed out by [9] and the references therein. However, there exist situations where the use of a delay in the controller may lead to closed-loop stability as remarked in [1], where a simple oscillator is stabilized by means of a positive delayed output feedback. This idea opens a new and interesting perspective in using delays as control parameters in order to get a desired behavior under appropriate assumptions; see [20].

In the spirit of the above observations, the works developed by $[4,5]$ have deeply explored such ideas, where, for a general retarded linear time-invariant system with commensurate delays, the authors have first fully characterized the stability properties of such a systems by finding a set of critical delay values, at which the system's characteristic quasi-polynomial has critical zeros on the imaginary axis. Secondly, considering the delay as a parameter and by adopting an operator based-approach the authors have expanded the solutions of the quasi-polynomial in terms of a Taylor (or Puiseux) series, allowing the characterization of the roots behavior as the delay varies around a critical delay value.

As discussed in [4], even in the case of a fixed delay, the testing of stability for a timedelay system is not a simple task. Such a difficulty arises from the fact that for delay systems the characteristic function is, in fact, a quasi-polynomial (as a consequence of the presence of delay) which always have an infinite number of characteristic roots (see, for instance, [10] and the references therein). In the retarded case, by using the appropriate continuity argument the problem can be reduced to the analysis on the critical characteristic roots on the imaginary axis. If the case when such roots

\footnotetext{
*Universidad Autónoma de San Luis Potosí (UASLP), Facultad de Ingeniería, Dr. Manuel Nava No.8, San Luis Potosí, S.L.P., México (jano.mtz.glz@gmail.com,cerfranfer@gmail.com,lfelixa@gmail.com).

${ }^{\dagger}$ Laboratoire des Signaux et Systèmes (L2S, UMR CNRS 8506), CNRS-CentraleSupélecUniversity Paris-Sud, 3, rue Joliot Curie, 91192, Gif-sur-Yvette, France (Silviu.Niculescu@l2s.centralesupelec.fr, jano.mtz.glz@gmail.com).

${ }^{\ddagger}$ Department of Electronic Engineering, City University of Hong Kong, Hong Kong, China (jichen@cityu.edu.hk).
} 
are simple is completely understood and fully characterized, the case of multiple characteristic roots need a deeper understanding. This paper focuses on this last problem.

By means of the Weierstrass Preparation Theorem [18] the complexity of the analysis of the system's characteristic function can be reduced to analyze some algebraic properties of a given polynomial (known as Weierstrass polynomial) with degree equal to the multiplicity of the critical zero. Furthermore, it is well known that such Weierstrass polynomial (for further details, see [11]) preserves the full information concerning the stability behavior. Such an approach has been adopted by [2], where by means of the calculus of residues (see, for instance, [21]), the authors have proposed an analytical method to construct such a polynomial. It is worth mentioning that the corresponding contribution does not focus on deriving an analytical characterization of the solutions or any characterization of the local behavior of the characteristic roots with respect to the delay parameter variation. Next, another important contribution in this direction is proposed by [13], where the authors found a explicit formula (see, for instance, Theorem 4) to characterize the local behavior for the first-order terms in the case of a double characteristic root. The asymptotic behavior of multiple critical roots has also been considered by $[17,16]$, where, instead of computing the Weierstrass polynomial, the authors derived the Puiseux series expansion of first-order by a direct application of the Newton diagram procedure. Their results allow computations in an efficient way. Finally, more recently, in [15], the asymptotic behavior analysis of critical solutions with respect to a infinitely many critical delays (including the case of one delay) has been considered where, in particular, the authors solve the general invariance property treated under some appropriate constraints in [13].

From the discussion above, it appears that the problem (asymptotic behavior characterization) received a lot of attention in the open literature during the last decade but is far from being closed. This paper intends to attack the problem under a different angle. In essence, the analysis is based on the Weierstrass Preparation Theorem as well as on the Puiseux Theorem [24] which in conjunction with the Newton diagram [3], allow proposing some simple algorithm to construct the Puiseux series for multiple imaginary roots of a given quasi-polynomial. This approach leads to a deeper understanding of the asymptotic behavior of critical zeros but also to determine the analyticity as well as the splitting properties of its branches, leading to a full characterization of its local behavior. The corresponding critical roots depict completely regular splitting, regular splitting and non regular splitting properties (for further details, see, subsection 3.2). Such a classification will be shown to be quite simple, and extremely useful in analyzing the local behavior as well as to study the related stability properties. Finally, a higher-order analysis is also presented, which is necessary to give conditions in order to determine the nature of the series expansion as a Taylor or Puiseux series. For the latter, by adopting similar ideas than those developed by $[19,4,5]$ we give conditions to determine the crossing directions.

The organization of this work is given as follows: section II introduces some preliminary results and the problem formulation. Section III is devoted to the main results. More precisely, an algorithm is proposed to compute the Puiseux (or Taylor) series expansion of a given solution. In addition, this section also presents the splitting properties and crossing directions and proposes a classification for the critical solutions. Finally, section IV includes some numerical examples illustrating the proposed results. The contribution ends with some concluding remarks.

Notations: In what follows, the following notations will be adopted: $\mathbb{C}$ (RHP, LHP) is the set of complex numbers (with strictly positive and strictly negative real 
parts), $\boldsymbol{i}:=\sqrt{-1}$. For $z \in \mathbb{C}, \arg (z) \in[0,2 \pi), \Re(z)(\Im(z))$ denote the argument, real (imaginary) part of $z$, respectively. Next, $\mathbb{R}_{+}$denotes the set of positive real values. The order of a power series $f(x, y)=\sum_{i, j} a_{i, j} x^{i} y^{j}$ will be denoted by ord $(f)$ and defined as the smallest number $n=i+j$ such that $a_{i, j} \neq 0$. The order of the power series $f$ with respect to the variable $x$, will be denoted by $\operatorname{ord}_{x}(f)$ and defined similarly. Finally, given two polynomials $f(z)=\sum_{j=0}^{n} a_{n-j} z^{j}$ and $g(z)=$ $\sum_{j=0}^{m} b_{m-j} z^{j}$, the resultant of $f$ and $g$ is defined as

$$
\mathcal{R}(f, g):=\operatorname{det}\left[\begin{array}{cccccccc}
a_{0} & a_{1} & a_{2} & \cdots & a_{n} & & & \\
& a_{0} & a_{1} & \cdots & \cdots & a_{n} & & \\
& & \ddots & \ddots & \ddots & & \ddots & \\
& & & a_{0} & a_{1} & \cdots & \cdots & a_{n} \\
b_{0} & b_{1} & b_{2} & \cdots & b_{m} & & & \\
& b_{0} & b_{1} & \cdots & \cdots & b_{m} & & \\
& & \ddots & \ddots & \ddots & & \ddots & \\
& & & b_{0} & b_{1} & \cdots & \cdots & b_{m}
\end{array}\right] .
$$

REMARK 1.1. One of the properties of this resultant (see, for instance, [24, 3]) is that $\mathcal{R}(f, g) \equiv 0$ if and only if $f$ and $g$ have common nonconstant factors.

\section{Preliminaries and Problem Formulation.}

2.1. Preliminary Results. Consider a retarded linear time-invariant system described in the state-space form as

$$
\dot{x}(t)=A_{0} x(t)+\sum_{k=1}^{q} A_{k} x(t-k \tau), \quad \tau \geq 0,
$$

or by the differential-difference equation,

$$
y^{(n)}(t)+\sum_{\ell=0}^{n-1} \sum_{k=0}^{q} a_{k \ell} y^{(\ell)}(t-k \tau)=0, \quad \tau \geq 0,
$$

under appropriate initial conditions. Let $f: \mathbb{C} \times \mathbb{R}_{+} \rightarrow \mathbb{C}$ be the corresponding quasi-polynomial given by

$$
f(s, \tau)=\operatorname{det}\left(s I-\sum_{k=0}^{q} A_{k} e^{-s k \tau}\right)=\sum_{k=0}^{q} p_{k}(s) e^{-k \tau s}, \quad \tau \geq 0,
$$

where the polynomials $p_{k}$ are given by

$$
p_{0}(s)=s^{n}+\sum_{\ell=0}^{n-1} a_{0 \ell} s^{\ell}, \quad p_{k}(s)=\sum_{\ell=0}^{n-1} a_{k \ell} s^{\ell}, \quad k=1, \ldots, q .
$$

The corresponding critical delay values for the imaginary roots can be computed by following the results presented in $[5,6]$.

2.1.1. Local Properties of Analytic functions. It it possible to reduce the analytic properties of $f(x, y)$ to algebraic properties. To this purpose, let us consider the following result. 
Theorem 2.1 (Weierstrass Preparation Theorem [18, 21]). Let $f(z, \boldsymbol{p})$ be an analytic function vanishing at the singular point $z_{0} \in \mathbb{C}, \boldsymbol{p}_{0} \in \mathbb{C}^{n}$, where $z=z_{0}$ is an $m$-multiple root of the equation $f(z, \boldsymbol{p})=0$, i.e.,

$$
f\left(z_{0}, \boldsymbol{p}_{0}\right)=\left.\frac{\partial f}{\partial z}\right|_{\left(z_{0}, \boldsymbol{p}_{0}\right)}=\cdots=\left.\frac{\partial^{m-1} f}{\partial z^{m-1}}\right|_{\left(z_{0}, \boldsymbol{p}_{0}\right)}=0,\left.\quad \frac{\partial^{m} f}{\partial z^{m}}\right|_{\left(z_{0}, \boldsymbol{p}_{0}\right)} \neq 0 .
$$

Then, there exist a neighborhood $U_{0} \subset \mathbb{C}^{n+1}$ of the point $\left(z_{0}, \boldsymbol{p}_{0}\right) \in \mathbb{C}^{n+1}$ in which the function $f(z, \boldsymbol{p})$ can be expressed as

$$
f(z, \boldsymbol{p})=W(z, \boldsymbol{p}) b(z, \boldsymbol{p})
$$

where

$$
W(z, \boldsymbol{p})=\left(z-z_{0}\right)^{m}+w_{m-1}(\boldsymbol{p})\left(z-z_{0}\right)^{m-1}+\cdots+w_{0}(\boldsymbol{p})
$$

and $w_{0}(\boldsymbol{p}), \ldots, w_{m-1}(\boldsymbol{p}), b(z, \boldsymbol{p})$ are analytic functions uniquely defined by the function $f(z, \boldsymbol{p})$ and $w_{i}\left(\boldsymbol{p}_{0}\right)=0, b\left(z_{0}, \boldsymbol{p}_{0}\right) \neq 0$.

REMARK 2.2. The analytic function $W(z, \boldsymbol{p})$ is known as the Weierstrass polynomial (for further details on Weierstrass polynomials, see, for instance, [11, 21]).

REMARK 2.3. It can be seen from Theorem 2.1 that since $b(z, \boldsymbol{p})$ is an holomorphic non vanishing function at $(0, \mathbf{0})$ then there must exist some neighborhood $\Omega(0, \mathbf{0}) \subset \mathbb{C}^{n+1}$ at which $b(z, \boldsymbol{p})$ preserves the same property. Hence, based on this observation we can ensure that the root-locus of a given quasi-polynomial $f$ in the neighborhood $\Omega$ will be the same as the root-locus of $W(z, \boldsymbol{p})$.

2.1.2. Newton Diagram Method. Given a known solution $\left(z_{0}, \boldsymbol{p}_{0}\right)$ of $f(z, \boldsymbol{p})$, the local behavior of the solution $z(\boldsymbol{p})$ in the neighborhood $\mathbb{C}^{n}$ of $\boldsymbol{p}$ can be obtained by means of the Newton diagram method. Thus, in order to use such a procedure, let us introduce the following notation (for more details, see, for instance, [22]). Let $f(x, y)$ be a pseudo-polynomial in $y$, i.e.,

$$
f(x, y)=\sum_{k=0}^{n} a_{k}(x) y^{k},
$$

where the corresponding coefficients are given by

$$
a_{k}(x)=x^{\rho_{k}} \sum_{r=0}^{\infty} a_{r k} x^{r / q},
$$

where $a_{r k}$ are complex numbers, $x$ and $y$ are complex variables, $\rho_{k}$ are non-negative rational numbers, $q$ is an arbitrary natural number, $a_{n}(x) \not \equiv 0$, and $a_{0}(x) \not \equiv 0$.

Since by simple translation, any point on a curve can be moved to the origin, we will consider expansions of the solution of $(5) f(x, y)=0$ around the origin, in the following form

$$
y(x)=y_{\epsilon_{1}} x^{\epsilon_{1}}+y_{\epsilon_{2}} x^{\epsilon_{2}}+y_{\epsilon_{3}} x^{\epsilon_{3}}+\cdots,
$$

where $\epsilon_{1}<\epsilon_{2}<\epsilon_{3}<\cdots, y_{\epsilon_{1}} \neq 0$. To determine the possible values of $\epsilon_{1}, y_{\epsilon_{1}}, \epsilon_{2}, y_{\epsilon_{2}}$, $\ldots$, it is necessary to consider the Newton diagram. 
Definition 2.4 (Newton's diagram and polygon). Given a pseudo-polynomial of the form (5) with coefficients given by (6), plot $\rho_{k}$ versus $k$ for $k$ for $k=0,1, \ldots, n$ (if $a_{k}(\cdot) \equiv 0$, the corresponding point is disregarded). Denote each of these points by $\pi_{k}=\left(k, \rho_{k}\right)$ and let

$$
\Pi=\left\{\pi_{k}: a_{k}(\cdot) \neq 0\right\}
$$

be the set of all plotted points. Then, the set $\Pi$ will be called the Newton diagram, and the Newton polygon associated with $f(x, y)$ will be given by the lower boundary of the convex hull of the set $\Pi$.

For a given pseudo-polynomial $f(x, y)$, Figure 1 simply illustrates Definition 2.4.

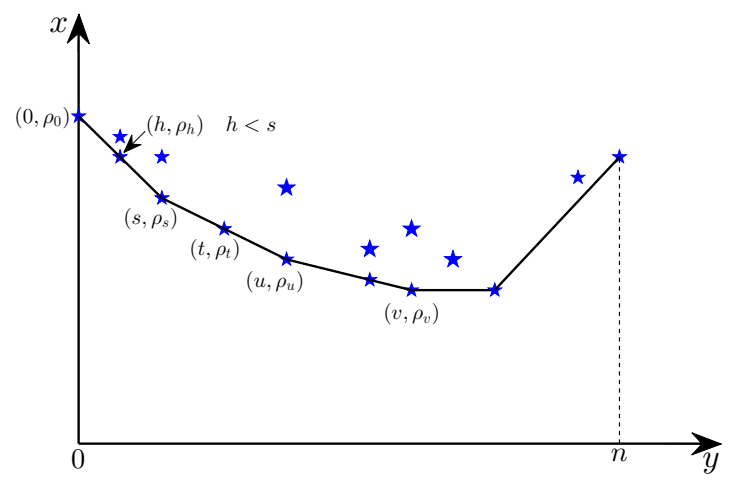

Figure 1. The Newton Diagram for the pseudo-polynomial $f(x, y)$ given in (5).

Theorem 2.5 (Puiseux Theorem, $[24,3]$ ). The equation $f(x, y)=0$, with $f$ given in formal power series such that $f(0,0)=0$, possess at least one solution in power series of the form

$$
x=t^{q}, \quad y=\sum_{i=1}^{\infty} c_{i} t^{i}, \quad q \in \mathbb{N} .
$$

2.2. Problem Formulation. The essential problem addressed in this work concerns the development of an analytical and efficient method to find explicitly the solutions of the equation $f(s, \tau)=0$ around some critical values. More precisely, in this paper, we will focus on the following problems:

(i) for a given quasi-polynomial $f$ and a known $m$-multiple solution $\left(i \omega^{*}, \tau^{*}\right) \in$ $\mathbb{C} \times \mathbb{R}_{+}$, find for the solution $s(\tau)$ the first coefficients of its Puiseux series expansion, i.e., compute $\gamma_{1}, p$ and $q$ such that

$$
s(\tau)=\boldsymbol{i} \omega^{*}+\gamma_{1}\left(\tau-\tau^{*}\right)^{\frac{p}{q}}+o\left(\left|\tau-\tau^{*}\right|^{\frac{p}{q}}\right), \quad \text { with } q \leq m, p \in \mathbb{Z} ;
$$

(ii) under assumption (i), give conditions on $f(s, \tau)$ which describes the splitting properties of its solutions $s(\tau)$ : regular splitting, completely regular splitting and nonregular splitting;

(iii) find the stability crossing directions, that is, determine whether the solution $s(\tau)$ enter to the right half-plane (or to the left half-plane) for $\tau>\tau^{*}$; 
(iv) determine the nature of the series expansion for the solution $s(\tau)$ in order to be able to identify if this solution will be expanded as a Taylor series

$$
s(\tau)=i \omega^{*}+c_{1}\left(\tau-\tau^{*}\right)+c_{2}\left(\tau-\tau^{*}\right)^{2} \cdots
$$

or as a Puiseux series,

$$
s(\tau)=i \omega^{*}+c_{1}\left(\tau-\tau^{*}\right)^{1 / m}+c_{2}\left(\tau-\tau^{*}\right)^{2 / m}+\cdots .
$$

\section{Main Results.}

3.1. Asymptotic zero behavior characterization. The asymptotic behavior of the critical zeros of the quasi-polynomial $f(s, \tau)$ will be performed by means of the Newton diagram procedure. To this end, since any critical solution $\left(s^{*}, \tau^{*}\right)$ can always be translated to the origin by appropriate shifts $s \mapsto s-s^{*}, \tau \mapsto \tau-\tau^{*}$, hereinafter we will assume that $\left(s^{*}, \tau^{*}\right)=(0,0)$. Hence, for a $m$-multiple root $s=0$ of $f$ at $\tau=0$, according to the Weierstrass Preparation Theorem we will have that

$$
f(s, \tau)=\left(s^{m}+w_{m-1}(\tau) s^{m-1}+\cdots+w_{0}(\tau)\right) b(s, \tau) .
$$

Now, with the aim of avoiding unnecessary computations, the following notations will be useful. For $i \in\{0,1, \ldots, m-1\}$, let $n_{i} \in \mathbb{N}$ denote the first nonzero partial derivatives in $(s, \tau)$ of $f$ at $(0,0)$, such that the following relations hold:

$$
f(0,0)=\left.\frac{\partial^{i} f}{\partial s^{i}}\right|_{(0,0)}=\cdots=\left.\frac{\partial^{i+n_{i}-1} f}{\partial s^{i} \partial \tau^{n_{i}-1}}\right|_{(0,0)}=0,\left.\quad \frac{\partial^{i+n_{i}} f}{\partial s^{i} \partial \tau^{n_{i}}}\right|_{(0,0)} \neq 0 .
$$

Even though the following result is a straightforward application of the Taylor series, it will be extremely useful in what follows.

LEMMA 3.1. Let $s=0$ be a m-multiple root at $\tau=0$ of the quasi-polynomial $f(s, \tau)$, and let $f=W b$ be defined as in (4). Then, the following statements hold:

(i) the first nonzero partial derivatives with respect to $\tau$ are given by

$$
\left.\frac{\partial^{n_{0}} f}{\partial \tau^{n_{0}}}\right|_{(0,0)}=\left.\frac{\partial^{n_{0}} w_{0}}{\partial \tau^{n_{0}}}\right|_{(0,0)} b(0,0)
$$

(ii) the first nonzero partial derivatives with respect to $s$ and $\tau$ for $i=1, \ldots, m-1$ are given by

$$
\left.\frac{\partial^{i+n_{i}} f}{\partial \tau^{n_{i}} \partial s^{i}}\right|_{(0,0)}=\left.\sum_{j=0}^{i}\left[j !\left(\begin{array}{c}
i \\
j
\end{array}\right) \sum_{k=0}^{n_{i}}\left(\begin{array}{c}
n_{i} \\
k
\end{array}\right) \frac{\partial^{n_{i}-k} w_{j}}{\partial \tau^{n_{i}-k}} \frac{\partial^{i-j+k} b}{\partial \tau^{k} \partial s^{i-j}}\right]\right|_{(0,0)} ;
$$

and,

(iii) the $m$-derivatives with respect to $s$ at the multiple critical point satisfy

$$
\left.\frac{\partial^{m} f}{\partial s^{m}}\right|_{(0,0)}=m ! b(0,0)
$$

Proof. According to Theorem 2.1, we have that $f$ admits the representation given in (8). Based on the previous observations, let us consider in the remaining $n_{i}$ as given in $(9)$. 
(i) It is not difficult to see that the first $r$-partial derivatives with respect to $\tau$ are given by

$$
\frac{\partial^{r} f}{\partial \tau^{r}}=\sum_{j=0}^{r}\left(\begin{array}{l}
r \\
j
\end{array}\right) \frac{\partial^{r-j} w_{0}}{\partial \tau^{r-j}} \frac{\partial^{j} b}{\partial \tau^{j}}+\frac{\partial^{r}}{\partial \tau^{r}} \sum_{j=1}^{m-1} w_{j} s^{j} b .
$$

Now, observe that

$$
\left.\frac{\partial^{r}}{\partial \tau^{r}} \sum_{j=1}^{m-1} w_{j} s^{j} b\right|_{(0,0)} \equiv 0 \forall r \geq 0, \quad \text { and }\left.\quad \sum_{j=0}^{r}\left(\begin{array}{l}
r \\
j
\end{array}\right) \frac{\partial^{r-j} w_{0}}{\partial \tau^{r-j}} \frac{\partial^{j} b}{\partial \tau^{j}}\right|_{(0,0)} \equiv 0 \forall r<n_{0} .
$$

Thus, it is clear that for $r=n_{0}$ and $(s, \tau)=(0,0)$, we get the desired result.

(ii) Following similar steps to those presented in (i), one gets

$$
\begin{aligned}
\frac{\partial^{r+n} f}{\partial \tau^{n} \partial s^{r}}= & \sum_{j=0}^{r}\left[j !\left(\begin{array}{l}
r \\
j
\end{array}\right) \sum_{k=0}^{n}\left(\begin{array}{l}
n \\
k
\end{array}\right) \frac{\partial^{n-k} w_{j}}{\partial \tau^{n-k}} \frac{\partial^{r-j+k} b}{\partial \tau^{k} \partial s^{r-j}}\right]+ \\
& \frac{\partial^{n}}{\partial \tau^{n}} \sum_{j=0}^{r}\left(\begin{array}{l}
r \\
j
\end{array}\right)\left[\sum_{k=j+1}^{m-1} \frac{k !}{(k-j) !} w_{k} s^{k-j}+\frac{m !}{(m-j) !} s^{m-j}\right] \frac{\partial^{r-j} b}{\partial s^{r-j}} .
\end{aligned}
$$

Hence, for $r=i, n=n_{i}$, and by evaluating at the critical point $(0,0)$ we have

$$
\left.\frac{\partial^{i+n_{i}} f}{\partial \tau^{n_{i}} \partial s^{i}}\right|_{(0,0)}=\left.\sum_{j=0}^{i}\left[j !\left(\begin{array}{c}
i \\
j
\end{array}\right) \sum_{k=0}^{n_{i}}\left(\begin{array}{c}
n_{i} \\
k
\end{array}\right) \frac{\partial^{n_{i}-k} w_{j}}{\partial \tau^{n_{i}-k}} \frac{\partial^{i-j+k} b}{\partial \tau^{k} \partial s^{i-j}}\right]\right|_{(0,0)} .
$$

(iii) The first nonzero partial derivatives in $s$ of $f$ are given by

$$
\frac{\partial^{m} f}{\partial s^{m}}=\sum_{j=0}^{m-1} w_{j}\left[\frac{\partial^{m} b}{\partial s^{m}}+j m \sum_{k=1}^{j} \frac{\partial^{m-k} b}{\partial s^{m-k}} s^{j-k}\right]+m \sum_{k=1}^{m-1}(k+1) ! \frac{\partial^{m-k} b}{\partial s^{m-k}} s^{m-k}+\frac{\partial^{m} b}{\partial s^{m}} s^{m}+m ! b .
$$

By evaluating it at the origin, it becomes $m ! b(0,0)$.

Proposition 3.2. Let $s=0$ be a $m$-multiple root at $\tau=0$ of the quasi-polynomial $f(s, \tau)$, and assume that $n_{0}<\infty$. Then, the Newton diagram of $f$ at $(0,0)$ is given by $\Pi=\left\{\left(0, n_{0}\right), \ldots,\left(m-1, n_{m-1}\right),(m, 0)\right\}$.

Proof. First note from the Weierstrass Preparation Theorem, the definition of $n_{i}$ and Lemma 3.1 that the Newton diagram of $f$ has the end points at $\left(0, n_{0}\right)$ and $(m, 0)$. Moreover, around the singular point $(0,0), f$ can be written as

$$
f(s, \tau)=\sum_{i=0}^{\infty}\left\{\frac{1}{i !} \sum_{j=i}^{\infty}\left(\begin{array}{l}
j \\
i
\end{array}\right) \frac{\partial^{j} f}{\partial s^{i} \partial \tau^{j-i}} \tau^{j-i}\right\} s^{i},
$$

or equivalently as

$$
f(s, \tau)=\sum_{i=0}^{m-1}\left\{\frac{1}{i !} \sum_{j=n_{i}+1}^{\infty}\left(\begin{array}{l}
j \\
i
\end{array}\right) \frac{\partial^{j} f}{\partial s^{i} \partial \tau^{j-i}} \tau^{j-i}\right\} s^{i}+\sum_{i=m}^{\infty}\left\{\frac{1}{i !} \sum_{j=i}^{\infty}\left(\begin{array}{l}
j \\
i
\end{array}\right) \frac{\partial^{j} f}{\partial s^{i} \partial \tau^{j-i}} \tau^{j-i}\right\} s^{i} .
$$

Hence, from Lemma 3.1-(ii), the remaining $(m-2)$-points of the Newton diagram of $f$ are given by the set $\left\{\left(1, n_{1}\right) \ldots,\left(m-1, n_{m-1}\right)\right\}$, which concludes the proof. 
EXAMPLE 3.1. In order to illustrate the previous result, let us consider the following quasi-polynomial (borrowed from [2]):

$$
f(s, \tau)=-\left(\frac{\pi}{2} s^{5}+\frac{\pi}{2} s^{3}+s^{2}\right)+\left(\frac{\pi}{2} s^{3}-s^{2}+\frac{\pi}{2} s+1\right) e^{-s \tau}+e^{-2 s \tau},
$$

with $s=i$ a multiple root at $\tau=\pi$ of multiplicity $m=3$. First, let us derive the constants $n_{i}$ considered in (9):

$$
\begin{gathered}
\left.\frac{\partial f}{\partial \tau}\right|_{(\boldsymbol{i}, \pi)}=0,\left.\frac{\partial^{2} f}{\partial \tau^{2}}\right|_{(\boldsymbol{i}, \pi)}=-2 \Rightarrow n_{0}=2,\left.\quad \frac{\partial^{2} f}{\partial s \partial \tau}\right|_{(\boldsymbol{i}, \pi)}=2+\boldsymbol{i} \pi, \Rightarrow n_{1}=1, \\
\left.\frac{\partial^{3} f}{\partial s^{2} \partial \tau}\right|_{(\boldsymbol{i}, \pi)}=-\left(5 \pi+\boldsymbol{i}\left(4 \pi^{2}+6\right)\right) \Rightarrow n_{2}=1,\left.\quad \frac{\partial^{3} f}{\partial s^{3}}\right|_{(\boldsymbol{i}, \pi)}=-3 \pi\left(-6-5 \boldsymbol{i} \pi+\pi^{2}\right) .
\end{gathered}
$$

Summarizing, we have $\left(n_{0}, n_{1}, n_{2}\right)=(2,1,1)$. Thus, according to Proposition 3.2 , we have $\Pi=\{(0,2),(1,1),(2,1),(3,0)\}$.

Under some appropriate considerations, the leading terms of the Weierstrass polynomial can be derived by means of the Taylor expansion of $f(s, \tau)$. In other words, the first nonzero partial derivatives of $f$ evaluated at $(0,0)$ can determine the first terms of the Weierstrass polynomial; we formalize this discussion through the following result.

Proposition 3.3. Let $n_{i}<\infty$ be defined as in (9), such that it satisfies

$$
n_{0}>n_{1}>\cdots>n_{m-1} \text {. }
$$

Then, the coefficients $w_{i}(\tau)$ of the associated Weierstrass polynomial $W$ have order ord $\left(w_{i}(\tau)\right)=n_{i}$. Moreover, the leading terms are given by

$$
w_{i}(\tau)=\left(\left.\frac{m !}{\left.i ! n_{i} ! \frac{\partial^{m} f}{\partial s^{m}}\right|_{(0,0)}} \frac{\partial^{i+n_{i}} f}{\partial \tau^{n_{i}} \partial s^{i}}\right|_{(0,0)}\right) \tau^{n_{i}}+o\left(\tau^{n_{i}}\right), i=0,1, \ldots, m-1 .
$$

Proof. According to Theorem 2.1, we have that

$$
f(s, \tau)=W(s, \tau) b(s, \tau)
$$

where $b(0,0) \neq 0$. Now, by applying Lemma 3.1-(ii) to the above expression and from the fact that $n_{0}>\cdots>n_{i}$, we deduce that:

$$
\begin{aligned}
\left.\frac{\partial^{i+n_{i}} f}{\partial \tau^{n_{i}} \partial s^{i}}\right|_{(0,0)} & =\left.i ! \sum_{k=0}^{n_{i}}\left(\begin{array}{c}
n_{i} \\
k
\end{array}\right) \frac{\partial^{n_{i}-k} w_{i}}{\partial \tau^{n_{i}-k}} \frac{\partial^{k} b}{\partial \tau^{k}}\right|_{(0,0)} \\
\left.\Rightarrow(b(0,0) i !) \frac{\partial^{n_{i}} w_{i}}{\partial \tau^{n_{i}}}\right|_{(0,0)} & =\left.\frac{\partial^{i+n_{i}} f}{\partial \tau^{n_{i}} \partial s^{i}}\right|_{(0,0)} .
\end{aligned}
$$

Next, from (9) we have that $\operatorname{ord}_{\tau}\left(w_{i}\right) \equiv n_{i}$ and since we know from Theorem 2.1 that $w_{i}(\tau)$ are analytic functions, this implies that

$$
w_{i}(\tau)=w_{i, 0} \tau^{n_{i}}+o\left(\tau^{n_{i}}\right) .
$$

Then, it is clear to see from (13) and (14) that

$$
\left.\frac{\partial^{i+n_{i}} f}{\partial \tau^{n_{i}} \partial s^{i}}\right|_{(0,0)}=i ! n_{i} ! w_{i, 0} b(0,0) \text {. }
$$

Finally, from Lemma 3.1-(iii) we get the desired result. 
REMARK 3.4. Note that it is possible to have some $\kappa \in \mathbb{N}$ for which $n_{0}=n_{1}=$ $\cdots=n_{\kappa-1}=\infty$. Then, under this situation, the Newton diagram method cannot be applied directly. However, it is worth noting that since $w_{i}$ are analytic functions, the previous situation is equivalent to $w_{i}(\tau) \equiv 0$ for $0 \leq i \leq \kappa-1$. Hence, $f$ will be locally given by

$$
f(s, \tau)=s^{\kappa}\left[s^{m-\kappa}+w_{m-\kappa}(\tau) s^{m-\kappa-1}+\cdots+w_{\kappa}(\tau)\right] b(s, \tau) .
$$

Thus, there are $\kappa$-invariant solutions $s=0$ for all $\tau$ and $m-\kappa$ solutions of the form

$$
s_{i}(\tau)=\sum_{j=1}^{\infty} c_{j} \tau^{j / m_{i}},
$$

where $m_{i}<m$. Moreover, under this consideration the Newton polygon will be given by $\Pi=\left\{\left(\kappa, n_{\kappa}\right), \ldots,(m, 0)\right\}$. If such number $\kappa$ does not exist (i.e., if such situation does not happen), then $\kappa$ will be simply defined as $\kappa:=0$.

3.2. Splitting Properties. The main goal of this subsection is to explore some qualitative properties of the solutions $s(\tau)$ of the quasi-polynomial $f(s, \tau)$ around the $m$-multiple critical pair $(0,0)$. Hence, as discussed by [24], it is possible to characterize the root locus of $f$ by its branches. In fact, the equation $f(s, \tau)=0$ defines a solution curve $\mathcal{C} \in \mathbb{C}^{2}$ which is composed by the finite union of $r$-branches $s_{j}\left(\tau^{1 / m_{j}}\right)$; each of these branches can be expressed as a Puiseux series:

$$
s_{j \sigma}(\tau)=c_{j \sigma} \tau^{\frac{1}{m_{j}}}+o\left(|\tau|^{\frac{1}{m_{j}}}\right), \quad j=0, \ldots, r-1, \quad \sigma=1, \ldots, m_{j}
$$

where each branch has multiplicity $m_{j}$ such that $m=m_{1}+m_{2}+\cdots+m_{r}$. In the case when $r=1$, then $s_{j \sigma}$ and $c_{j \sigma}$ will be simply denoted by $s_{\sigma}$ and $c_{\sigma}$, respectively. We have the following.

DEFINITION 3.5. We say that there is a complete regular splitting (CRS) property of the solution $s^{*}=0$ at $\tau^{*}=0$ if $c_{j \sigma} \neq 0 \forall j$. For the regular splitting (RS) property, some of the coefficients $c_{j \sigma}$ for which $m_{j}=1$ may be equal to zero. In the remaining cases of the coefficient $c_{j \sigma}$ we say that nonregular splitting property is present.

REMARK 3.6. The above definition, illustrated in Figure 2, was inspired by the matrix case introduced in [14] (see also [12]).

The proposed approach to deal with the splitting properties is based on the Newton diagram method applied in conjunction with the Weierstrass polynomial and the Puiseux Theorem. To this end, we will use the definitions introduced in the previous sections, in particular the notion of Newton polygon $\Pi$.

Along these lines, based on the Newton procedure introduced in subsection 2.1.2 we propose Algorithm 1. 


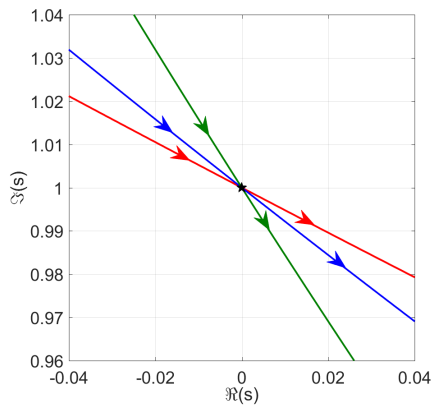

(a) Nonregular splitting

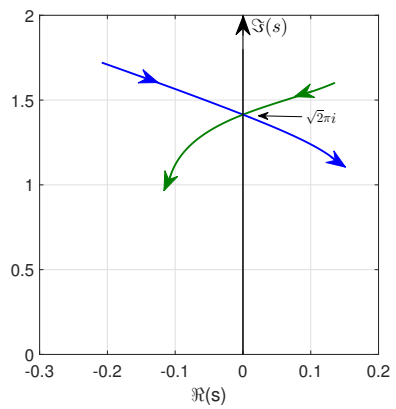

(b) Regular splitting

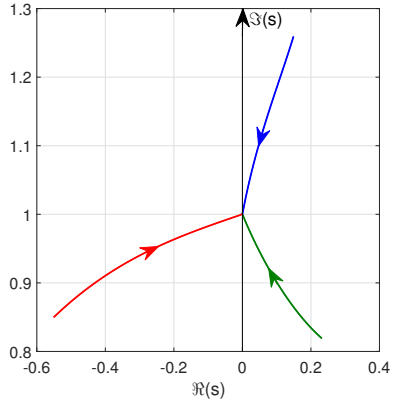

(c) Complete regular Splitting

Figure 2. Splitting properties.

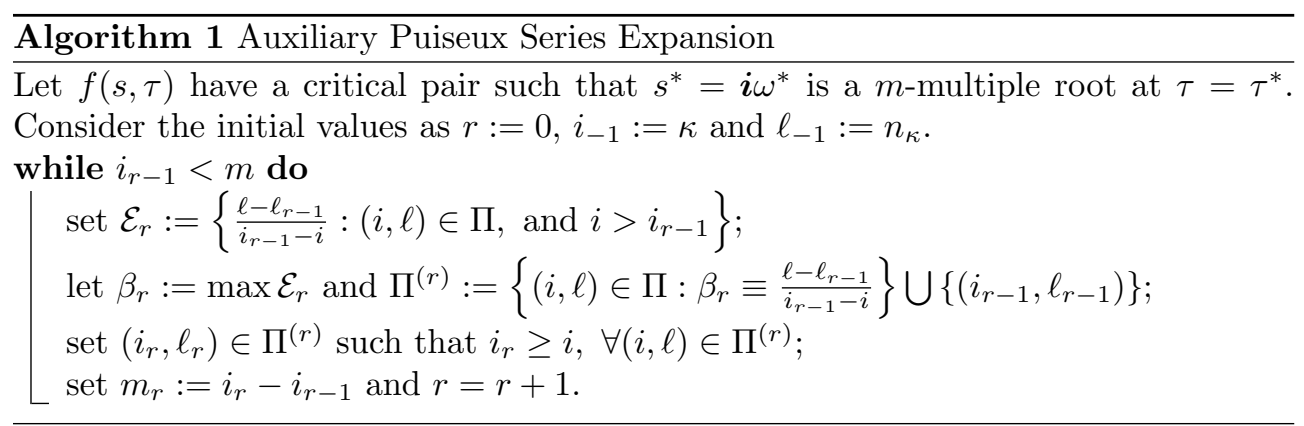

EXAMPLE 3.2. With the aim of illustrating the simplicity of the algorithm, let us apply it to the quasi-polynomial (11) (Example 3.1 above). First, observe that according to Remark 3.10 we have that $\kappa=0$, implying that the initial conditions for the Algorithm 1 are $\left(r, i_{-1}, j, k\right)=\left(0,0,0, n_{0}\right)$ and $\Pi$, where this last set has been determined in Example 3.1. Thus, for the first iteration we have that $\mathcal{E}_{0}=\left\{1, \frac{1}{2}, \frac{2}{3}\right\} \Rightarrow$ $\beta_{0}=1$ and $\Pi^{(0)}=\{(0,2),(1,1)\}$. According to step 3 we have $\left(i_{0}, \ell_{0}\right)=(1,1)$, which implies that the algorithm will end in the next iteration. Table 2 summarizes the results.

TABLE 1

Results summary for the quasi-polynomial (11).

\begin{tabular}{ll}
\hline Initial data & Algorithm output \\
\hline$m=3, n_{0}=2$ & $r=1, m_{0}=1, \beta_{0}=1$ \\
$\Pi=\{(0,2),(1,1),(2,1),(3,0)\}$ & $\Pi^{(0)}=\{(0,2),(1,1)\}$ \\
\cline { 2 - 2 } & $r=2, m_{1}=2, \beta_{1}=\frac{1}{2}$ \\
& $\Pi^{(1)}=\{(1,1),(3,0)\}$ \\
\hline
\end{tabular}

We have the following.

Proposition 3.7. Let $s^{*}=i \omega^{*}$ at $\tau=\tau^{*}$ be a m-multiple critical root of the quasi-polynomial $f(s, \tau)$. Assume that $r, \beta_{j},\left(i_{j}, \ell_{j}\right), m_{j}$ and $\Pi^{(j)}$, for $j=0,1, \ldots, r-$ 1 are given by Algorithm 1. Then, the following properties hold: 
(i) if $m_{j} \cdot \beta_{j} \equiv 1 \forall j \in\{0,1, \ldots, r-1\}$, then the solution $\left(i \omega^{*}, \tau^{*}\right)$ of $f(s, \tau)$ has the completely regular splitting property;

(ii) if some $\beta_{j}$ satisfies $m_{j} \cdot \beta_{j}>1$ for $m_{j}>1$, then non regular splitting property for the solution $\left(\boldsymbol{i} \omega^{*}, \tau^{*}\right)$ occur;

(iii) if the pairs $\left(m_{k}, \beta_{k}\right)$ that do not fulfill (i), satisfy the inequality $\beta_{k} \geq m_{k} \equiv 1$, then the solution $\left(i \omega^{*}, \tau^{*}\right)$ of $f(s, \tau)$ has the regular splitting property;

(iv) let $\Omega_{0}$ be a neighborhood of $(0,0) \in \mathbb{C}^{2}$, and assume that $\mathcal{R}\left(W, \frac{\partial}{\partial s} W\right) \neq 0 \forall s \in$ $\Omega_{0} \backslash\{(0,0)\}$. Then, there are $m$-different Puiseux series solutions $g_{i}\left(\tau^{\frac{1}{n_{i}}}\right)$ such that

$$
f(s, \tau)=\prod_{i=1}^{m}\left(s-g_{i}\left(\tau^{\frac{1}{n_{i}}}\right)\right) b(s, \tau),
$$

where $n_{i}$ is arranged in terms of $m_{j}$ as

$$
\begin{aligned}
& \underbrace{n_{1}=n_{2}=\cdots=n_{m_{1}}}_{n_{i_{1}}=m_{1}}, \underbrace{n_{m_{1}+1}=\cdots=n_{m_{2}}}_{n_{i_{2}}=m_{2}}, \cdots, \underbrace{n_{m_{1}+\cdots+m_{r-1}+1}=\cdots=n_{m_{1}+\cdots+m_{r}}}_{n_{i_{r}}=m_{r}} \\
& \text { with } \sum m_{i}=m .
\end{aligned}
$$

Proof. First of all, observe that $r$ in Algorithm 1 corresponds to the number of branches for the solution $\left(\boldsymbol{i} \omega^{*}, \tau^{*}\right)$ and $m_{j}$ the multiplicity associated to each branch. (i) In this case, we have that $\beta_{j}=\frac{1}{m_{j}}$; then from the Newton procedure we know that the rational numbers $\beta_{j}$ are associated to the first exponents in the solutions, since we have $r$ branches, thus the root locus of $f(s, \tau)$, is given by

$$
s_{j \sigma}(\tau)=c_{j \sigma} \tau^{\frac{1}{m_{j}}}+o\left(\tau^{\frac{1}{m_{j}}}\right), \quad j=0, \ldots, r-1 \quad \sigma=1, \ldots, m_{j}
$$

Since $c_{j \sigma}$ are related to the nonzero solution of a polynomial formed with the coefficients of the convex hull, clearly $c_{j \sigma} \neq 0$. Then, the solution $\left(i \omega^{*}, \tau^{*}\right)$ has the CRS property.

(ii)-(iii) These follow in similar lines those presented in (i).

(iv) (See [3]). This case can be stated by induction. To this end, from the Puiseux Theorem we known that there exists a Puiseux series $g_{1}\left(\tau^{1 / n_{i}}\right)$ such that $f\left(g_{1}, \tau\right)=0$; then the factor $s_{1}$ can be taken out such that $f=g_{1} f_{1}$. Assume now that the above factorization is valid for some $k \in \mathbb{N}$, i.e., the following relation holds:

$$
f(s, \tau)=g_{1}(\tau) \cdots g_{k}(\tau) f_{k}(s, \tau) .
$$

Then the quotient $f_{k}$ has order $m-k$ in $s$. Applying the induction hypothesis to $f_{k}$, then we get $m$ different factors $g_{i}$ such that:

$$
f(s, \tau)=g_{1}(\tau) \cdots g_{m}(\tau) f_{m}(s, \tau),
$$

where $f_{m}(s, \tau)$ has order $\operatorname{ord}_{\tau}\left(f_{m}\right)=0$.

Corollary 3.8. Consider the same hypothesis as in Proposition 3.7. Assume that $n_{0}=1$. Then at $\tau=\tau^{*}$ the $m$-roots of $f(s, \tau)$ have the CRS property; i.e., these roots can be expanded as

$$
s_{\sigma}(\tau)=\boldsymbol{i} \omega^{*}+c_{\sigma}\left(\tau-\tau^{*}\right)^{\frac{1}{m}}+o\left(\left|\tau-\tau^{*}\right|^{\frac{1}{m}}\right), \quad \text { for } \sigma=1,2, \ldots, m .
$$

Moreover, the following properties hold: 
(i) if $m=2$ and $\Re\left(c_{\sigma}\right) \neq 0$ with $\sigma \in\{1,2\}$, then for $\tau>\tau^{*}$ sufficiently close to $\tau^{*}$, one of the zeros $s_{\sigma}(\tau)$ will enter the RHP, whereas the other one will enter the LHP;

(ii) if $m>2$, then at least one of the zeros $s_{\sigma}(\tau)$ will enter the RHP.

3.3. Crossing Directions Characterization. As mentioned in the introduction, the Weierstrass polynomial will be our main tool to analyze the stability behavior of the critical characteristic roots. In the same spirit as [5], we have the following result.

Proposition 3.9. Let $n_{0}<\infty$ and $s^{*}=i \omega^{*}$ be a m-multiple root of $f(s, \tau)$ at $\tau=\tau^{*}$. Assume that $r, \beta_{j},\left(i_{j}, \ell_{j}\right), m_{j}$, and $\Pi^{(j)}$ for $j=0,1, \ldots, r-1$ are given by Algorithm 1. Then, at $\tau=\tau^{*}$ the $m$-zeros of $f(s, \tau)$ can be expanded as

$$
s_{j \sigma}(\tau)=i \omega^{*}+c_{j \sigma}\left(\tau-\tau^{*}\right)^{\beta_{j}}+o\left(\left|\tau-\tau^{*}\right|^{\beta_{j}}\right)
$$

for $j=0,1, \ldots, r-1, \sigma=1, \ldots, m_{j}$, and $m=m_{0}+\cdots+m_{r-1}$, where $c_{j \sigma}$ are roots of the polynomial $\mathcal{P}_{j}: \mathbb{C} \mapsto \mathbb{C}$,

$$
\mathcal{P}_{j}(z):=\sum_{k=i_{j-1}}^{i_{j}} a_{k, 0} z^{k-i_{j-1}}, \quad \text { s.t. }\left(k, \eta_{k}\right) \in \Pi^{(j)},
$$

where the coefficient $a_{k, 0} \in \mathbb{C}$ is given by

$$
a_{k, 0}=\left.\left(\frac{m !}{\left.k ! \eta_{k} ! \frac{\partial^{m} f}{\partial s^{m}}\right|_{(0,0)}}\right) \frac{\partial^{k+\eta_{k}} f}{\partial \tau^{\eta_{k}} \partial s^{k}}\right|_{(0,0)} .
$$

Furthermore, for $\tau>\tau^{*}$ sufficiently close to $\tau^{*}$, the zeros $s_{j \sigma}(\tau)$ will enter the right half-plane (or to the left half-plane) if

$$
\Re\left\{c_{j \sigma}\right\}>0(<0) .
$$

Proof. By taking into account the Newton procedure and applying Algorithm 1 to $f(s, \tau)$, it is clear to see that the solution $s_{j \sigma}$ can be expanded as in (17). Now, observe that proving that the coefficients $c_{j \sigma}$ are given by the solutions of $\mathcal{P}_{j}$ is equivalent to prove that the coefficients of the Weierstrass polynomial $W$ that lies on the Newton polygon are given by (19) modulus some constant factor. Thus, in order to show this fact we first notice that, according to Lemma 3.1-(i), $\operatorname{ord}_{\tau}\left(w_{0}\right)=n_{0}$. Moreover, from the definition of $n_{i}$, it is clear to see that $\operatorname{ord}_{\tau}\left(w_{i}\right)=n_{i}$. Next, since by Theorem 2.1 we have that $f=W b$ with $b(0,0) \neq 0$, and according to Lemma 3.1-(ii) we know that the first $\ell$ derivatives in $s$ and the $\tilde{n}$ derivatives in $\tau$ are given by

$$
\begin{aligned}
\frac{\partial^{\ell+\tilde{n}} f}{\partial \tau^{\tilde{n}} \partial s^{\ell}} & =\sum_{j=0}^{\ell}\left[j !\left(\begin{array}{l}
\ell \\
j
\end{array}\right) \sum_{k=0}^{\tilde{n}}\left(\begin{array}{l}
\tilde{n} \\
k
\end{array}\right) \frac{\partial^{\tilde{n}-k} w_{j}}{\partial \tau^{\tilde{n}-k}} \frac{\partial^{\ell-j+k} b}{\partial \tau^{k} \partial s^{\ell-j}}\right]+ \\
& \frac{\partial^{\tilde{n}}}{\partial \tau^{\tilde{n}}} \sum_{j=0}^{\ell}\left(\begin{array}{l}
\ell \\
j
\end{array}\right)\left[\sum_{k=j+1}^{m-1} \frac{k !}{(k-j) !} w_{k} s^{k-j}+\frac{m !}{(m-j) !} s^{m-j}\right] \frac{\partial^{\ell-j} b}{\partial s^{\ell-j}} .
\end{aligned}
$$


Let $v_{j}, \alpha_{v_{j}} \mathbb{N} \cup 0$ such that $\left(v_{j}, \alpha_{v_{j}}\right) \in \Pi^{(j)}$; then clearly these points will lie on the Newton polygon. Furthermore, such points satisfy the relation $\alpha_{v_{j}}>\alpha_{v_{k}}$ for $v_{j}<v_{k}$. Therefore, by taking $\ell=v_{j}$ and $\tilde{n}=\alpha_{v_{j}}$ in (21) it is clear to see from (13) and (14) that

$$
\left.\frac{\partial^{v_{j}+\alpha_{v_{j}}} f}{\partial \tau^{\alpha_{v_{j}}} \partial s^{v_{j}}}\right|_{(0,0)}=v_{j} ! \alpha_{v_{j}} ! w_{v_{j}, 0} b(0,0)
$$

Then, (19) follows by noting that $k=v_{j}$ and $\eta_{k}=\alpha_{v_{j}}$. Finally, the direction of crossing follows straightforwardly by condition (20).

REMARK 3.10. It is worth mentioning that in the above result, it is not necessary to assume that $n_{0}<\infty$ (i.e., $\kappa \neq 0$ ). In fact, in order to relax such an assumption, i.e., to consider $\kappa>0$, we have just assume that the $j$-index will take values in the set $\{\kappa, \kappa+1, \ldots, r-1\}$ and that the solution $s_{j \sigma}$ will be arranged in $(r-\kappa)$-branches of Puiseux series.

3.4. Higher-Order Analysis. In some situations, the first-order expansion does not give enough information to analyze the stability of a given solution. Such situations occur when (20) does not hold, that is, when the coefficient of the first-order term is purely imaginary. Thus, in order to cope with such a case study and inspired by the results developed by [23], in the following, we will consider a higher-order analysis.

Let $\beta_{j},\left(i_{j}, \ell_{j}\right) \in \Pi^{(j)}$ be given by Algorithm 1. It is not difficult to see that, in order to compute higher-order terms for the solution $s_{j \sigma}(\tau)$, we can make use of the change of variables $s \mapsto \tau^{\beta_{j}}\left(c_{j \sigma}+s_{1}\right)$ in $f(s, \tau)$ to get the function $f_{1}\left(s_{1}, \tau\right)$, and repeat the same procedure presented in the previous section for this function $f_{1}$. In this vein, the solution $s_{j \sigma}$ can be expressed as

$$
s_{j \sigma}(\tau)=c_{1} \tau^{\beta_{j}}+c_{2} \tau^{\beta_{j}+\beta_{j}^{(1)}}+c_{3} \tau^{\beta_{j}+\beta_{j}^{(1)}+\beta_{j}^{(2)}}+\cdots,
$$

where $\beta_{j}^{(1)}$ is the output of the Algorithm 1 for the function $f_{1}$, and so on. Now, according to the Newton procedure, we have that any arbitrary pair $\left(i, \eta_{i}\right)$ belonging to the set $\Pi^{(j)}$ must satisfy $\eta_{i}+i \beta_{j}=\nu_{j}$, with a fixed $\nu_{j} \in \mathbb{Q}$. Hence, in order to find some insights over $c_{2}$ and $\beta_{j}^{(1)}$ let us define the associated Weierstrass polynomial $W_{1}$ as $W_{1}\left(s_{1}, \tau\right):=\tau^{-\nu_{j}} W\left(\tau^{\beta_{j}}\left(c_{j \sigma}+s_{1}\right), \tau\right)$. Then, from all these facts, $W_{1}$ will be given as:

$W_{1}\left(s_{1}, \tau\right)=\tau^{-\nu_{j}}\left[\tau^{m \beta_{j}}\left(c_{1}+s_{1}\right)^{m}+w_{m-1}(\tau) \tau^{(m-1) \beta_{j}}\left(c_{1}+s_{1}\right)^{(m-1)}+\cdots+w_{0}(\tau)\right]$.

Now, since for the first-order term of the solution $s_{j \sigma}$, we only need to consider the terms on $\Pi^{(j)}$, according to Proposition 3.9, we have that the main coefficients of $W$ 
falling on $\Pi^{(j)}$ are denoted by $a_{l, 0}$, therefore $W_{1}$ can be rewritten as:

$$
\begin{aligned}
& W_{1}\left(s_{1}, \tau\right)=\underbrace{\tau^{-\nu_{j}} \sum_{l=i_{j-1}}^{i_{j}} a_{l, 0} \tau^{\eta_{l}+l \beta_{j}}\left(c_{1}+s_{1}\right)^{l}}_{=: W_{1}^{(m)}\left(s_{1}, \tau\right)}+ \\
& \tau^{-\nu_{j}\left[\sum_{l=i_{j-1}}^{i_{j}}\left(w_{l}-a_{l, 0} \tau^{\eta_{l}}\right) \tau^{l \beta_{j}}\left(c_{1}+s_{1}\right)^{l}+\sum_{h \notin \Pi(j)} w_{h} \tau^{h \beta_{j}}\left(c_{1}+s_{1}\right)^{h}\right]} .
\end{aligned}
$$

Taking into consideration that $\eta_{l}+l \beta_{j}=\nu_{j}$, the first summation in (23) can be reduced as follows:

$$
\begin{aligned}
\sum_{l=i_{j-1}}^{i_{j}} a_{l, 0} \tau^{n_{l}+l \beta_{j}}\left(c_{1}+s_{1}\right)^{l} & =\tau^{\nu_{j}}\left(c_{1}+s_{1}\right)^{i_{j-1}} \mathcal{P}_{j}\left(c_{1}+s_{1}\right), \\
& =\tau^{\nu_{j}}\left(c_{1}+s_{1}\right)^{i_{j-1}} s_{1}^{\mu} \psi\left(c_{1}+s_{1}\right),
\end{aligned}
$$

where, according to Proposition 3.9, $c_{1}$ is in general a $\mu$-multiple solution of $\mathcal{P}_{j}$ and $\psi\left(c_{1}\right) \neq 0$. Thus, $W_{1}^{(m)}$ will be expressed as

$$
W_{1}^{(m)}\left(s_{1}, \tau\right)=c_{1}^{i_{j-1}} \psi\left(c_{1}\right) s_{1}^{\mu}+\left(i_{j-1} c_{1}^{i_{j-1}-1} \psi\left(c_{1}\right)+c_{1}^{i_{j-1}} \psi^{\prime}\left(c_{1}\right)\right) s_{1}^{\mu+1}+\cdots .
$$

From the above discussion, it is clear to see that $\operatorname{ord}_{\tau}\left(W_{1}^{(m)}\right) \equiv 0$. Now, since $c_{1}^{i_{j-1}} \psi\left(c_{1}\right)=$ constant $\neq 0$, we have that $W_{1}$ can be expressed as:

$$
W_{1}\left(s_{1}, \tau\right)=w_{0}^{(1)}(\tau)+\cdots+w_{\mu}^{(1)}(\tau) s_{1}^{\mu}+\cdots+w_{m}^{(1)}(\tau) s_{1}^{m},
$$

with $\operatorname{ord}_{\tau}\left(w_{\mu}^{(1)}\right)=0$, implying that the end point for the Newton polygon of $W_{1}$ will be $(\mu, 0)$. Bearing in mind these facts, we have the following:

Proposition 3.11. Let $s^{*}=i \omega^{*}$ be a m-multiple root of $f(s, \tau)$ at $\tau=\tau^{*}$. Assume that $\beta_{j}$ and $m_{j}$ for $j=\kappa, \kappa+1, \ldots, r-1$ are given by the Algorithm 1 . If $\beta_{j}=1$, then the following statements hold:

(i) the equation $f(s, \tau)=0$ has $m_{j}-$ solutions of the form

$$
s_{j \sigma}(\tau)=\boldsymbol{i} \omega^{*}+c_{j \sigma}\left(\tau-\tau^{*}\right)+o\left(\left|\tau-\tau^{*}\right|\right), \quad \sigma=1, \ldots, m_{j},
$$

where $c_{j \sigma}$ is a root of the polynomial $\mathcal{P}_{j}$ defined in (18);

(ii) if $c_{j \sigma}$ is a simple root of $\mathcal{P}_{j}$ then, there are $m_{j}-$ solutions expanded as a Taylor series in the form

$$
s_{j \sigma}(\tau)=i \omega^{*}+c_{j \sigma}\left(\tau-\tau^{*}\right)+c_{j \sigma}^{(1)}\left(\tau-\tau^{*}\right)^{1+\beta_{j}^{(1)}}+\cdots,
$$

where $\beta_{j}^{(1)} \in \mathbb{N}$.

Proof. Let $m_{j}$ and $\beta_{j}$ be given by the Algorithm 1 .

(i) Since by hypothesis $\beta_{j}=1$, the condition (26) follows straightforwardly from the Newton procedure. 
(ii) Next, by hypothesis, we have that $c_{j \sigma}$ is a simple root of $\mathcal{P}_{j}$. Thus, using the same arguments as in the previous discussion, we have that $\mu \equiv 1$ implies that $(25)$ can be written as

$$
W_{1}\left(s_{1}, \tau\right)=w_{0}^{(1)}(\tau)+w_{1}^{(1)}(\tau) s_{1}+\cdots+w_{m}^{(1)}(\tau) s_{1}^{m},
$$

where $\operatorname{ord}_{\tau}\left(w_{1}^{(1)}\right)=0$. Now, since:

$$
w_{0}^{(1)}(\tau)=\tau^{-\nu_{j}}\left(w_{0}(\tau)+c_{j \sigma} w_{1}(\tau) \tau+\cdots+c_{j \sigma}^{m-1} w_{m-1}(\tau) \tau^{m-1}+c_{j \sigma}^{m} \tau^{m}\right)
$$

we have that $w_{0}^{(1)}(0) \equiv 0 \Rightarrow \operatorname{ord}_{\tau}\left(w_{0}^{(1)}\right) \geq 1$. Thus, since according to Theorem 2.1 we know that $w_{i}^{(1)}$ are analytic functions, and since $\operatorname{ord}_{\tau}\left(w_{0}^{(1)}\right) \geq 1$ one gets $\beta_{j}^{(1)} \in \mathbb{N}$.

Proposition 3.12. Let $s^{*}=i \omega^{*}$ be a m-multiple root of $f(s, \tau)$ at $\tau=\tau^{*}$. Assume that $\beta_{j}, m_{j}$ and $\left(i_{j}, \ell_{j}\right) \in \Pi^{(j)}$ for $j=0,1, \ldots, r-\kappa-1$ are given by the Algorithm 1. If $\beta_{j}=1 / m_{j}$, then $f(s, \tau)=0$ has $m_{j}-$ solutions given by

$$
s_{j \sigma}(\tau)=i \omega^{*}+c_{j} \Theta_{\sigma}\left(\tau-\tau^{*}\right)^{1 / m_{j}}+o\left(\left|\tau-\tau^{*}\right|^{1 / m_{j}}\right), \quad \sigma=1, \ldots, m_{j},
$$

where $\Theta_{\sigma}=\exp \left(i \frac{\theta_{j}+2 \pi(\sigma-1)}{m_{j}}\right), \theta_{j}=\arg \left(c_{j}^{m_{j}}\right)$ and $c_{j}=\left|a_{i_{j-1}, 0} / a_{i_{j}, 0}\right|^{1 / m_{j}}$.

Proof. The proof follows straightforwardly from Proposition 3.7.

Proposition 3.13. Let $s^{*}=i \omega^{*}$ be a $m$-multiple root of $f(s, \tau)$ at $\tau=\tau^{*}$. Let $\beta_{j}, m_{j}$ and $\left(i_{j}, \ell_{j}\right) \in \Pi^{(j)}$ for $j=0,1, \ldots, r-\kappa-1$ be given by the Algorithm 1. Assume that $\beta_{j}=1, c_{j \sigma}$ is a $m_{j}-$ multiple root of $\mathcal{P}_{j}$ and $\left.\frac{d^{\nu^{\nu}+1}}{\tau^{\nu_{j}+1}} f\left(c_{j \sigma} \tau, \tau\right)\right|_{\tau=0} \neq 0$, with $\nu_{j}=n_{i_{j-1}}+i_{j-1}$. Then, there are $m_{j}-$ solutions expanded as a Puiseux Series in the form

$s_{j \sigma}(\tau)=i \omega^{*}+c_{j \sigma}\left(\tau-\tau^{*}\right)+c_{j \sigma}^{(1)}\left(\tau-\tau^{*}\right)^{1+1 / m_{j}}+o\left(\left|\tau-\tau^{*}\right|^{1+1 / m_{j}}\right), \quad \sigma=1, \ldots, m_{j}$, where $c_{j \sigma}^{(1)}$ is a solution of the polynomial $\mathcal{P}_{j}$ given in (18), associated to $W_{1}$.

Proof. Let $m_{j}, \beta_{j}$ and $\left(i_{j}, \ell_{j}\right)$ be given by the Algorithm 1. First, note that since $\beta_{j} \equiv 1$ clearly the $m_{j}$-solution will be expanded as:

$$
s_{j \sigma}(\tau)=i \omega^{*}+c_{j \sigma}\left(\tau-\tau^{*}\right)+o\left(\left|\tau-\tau^{*}\right|\right), \quad \sigma=1, \ldots, m_{j} .
$$

Now, using similar arguments to the previous case study we have that $\mu \equiv m_{j}$, implies that (25) can be written as:

$$
W_{1}\left(s_{1}, \tau\right)=w_{0}^{(1)}(\tau)+\cdots+w_{m_{j}}^{(1)}(\tau) s_{1}^{m_{j}}+\cdots+w_{m}^{(1)}(\tau) s_{1}^{m},
$$

where $\operatorname{ord}_{\tau}\left(w_{m_{j}}^{(1)}\right)=0$. Thus, in order to have $\beta_{j}^{(1)}=1 / m_{j}, \operatorname{ord}_{\tau}\left(w_{0}^{(1)}\right)=1$ must be fulfilled. To see that such a condition hold, note that $w_{0}^{(1)}(\tau)=\tau^{-\nu_{j}} W\left(c_{j \sigma} \tau, \tau\right)$, and by Theorem 2.1, we have that $f=W b$, where ord $(b)=0 \Rightarrow \operatorname{ord}_{\tau}(W b)=\operatorname{ord}_{\tau}(W)$. Since

$$
\operatorname{ord}_{\tau}\left(w_{0}^{(1)}\right)=\left.1 \Leftrightarrow \frac{\partial}{\partial \tau} w_{0}^{(1)}\right|_{\tau=0} \neq 0
$$


we have that

$$
\left.\frac{\partial}{\partial \tau} w_{0}^{(1)}\right|_{\tau 0} \neq\left. 0 \Leftrightarrow \frac{\partial}{\partial \tau}\left(\tau^{-\nu_{j}} f\left(c_{j \sigma} \tau, \tau\right)\right)\right|_{\tau=0} \neq 0 .
$$

Finally, by noticing that

$$
\left.\frac{\partial}{\partial \tau}\left(\tau^{-\nu_{j}} f\left(c_{j \sigma} \tau, \tau\right)\right)\right|_{\tau=0} \neq\left. 0 \Leftrightarrow\left(\tau^{-\nu_{j}} f^{\prime}-\nu_{j} \tau^{-\nu_{j}-1} f\right)\right|_{\tau=0} \neq 0,
$$

and since,

$$
\left.\left(\tau^{-\nu_{j}} f^{\prime}-\nu_{j} \tau^{-\nu_{j}} f\right)\right|_{\tau=0} \neq\left. 0 \Leftrightarrow \frac{d^{\nu_{j}+1}}{\tau^{\nu_{j}+1}} f\left(c_{j \sigma} \tau, \tau\right)\right|_{\tau=0} \neq 0
$$

the proof is completed.

REMARK 3.14. Since the Weierstrass polynomial $W$ is derived using the quasipolynomial $f$, thus, instead of considering $W_{1}$ in Proposition 3.13 , it is possible to consider $f_{1}\left(s_{1}, \tau\right):=\tau^{-\nu_{j}} f\left(\tau^{\beta_{j}}\left(c_{j \sigma}+s_{1}\right), \tau\right)$.

4. Numerical Examples. In order to illustrate the effectiveness of the proposed methodology, in the sequel we propose several examples. Such examples have been performed by means of the software package DDE-BIFTOOL (see, for instance, $[8,7]$ ).

EXAMPLE 4.1. As a first example, let us consider the quasi-polynomial given in Example 3.1. Thus, Table 2 summarizes the results obtained by applying the Algorithm 1 in conjunction with Proposition 3.2 and Proposition 3.9.

\begin{tabular}{|c|c|c|}
\hline Initial Data & Algorithm Output & $\mathcal{Z}:=\left\{z \in \mathbb{C}: \mathcal{P}_{j}(z)=0\right\}$ \\
\hline \multirow{4}{*}{$\begin{array}{l}m=3, n_{0}=2 \\
\Pi=\{(0,2),(1,1),(2,1),(3,0)\}\end{array}$} & $r=0, m_{0}=1, \beta_{0}=1$ & $\mathcal{P}_{0}(z):=-\frac{4+2 \boldsymbol{i} \pi}{\pi\left(-6-5 \boldsymbol{i} \pi+\pi^{2}\right)} z+\frac{2}{\pi\left(-6-5 \boldsymbol{i} \pi+\pi^{2}\right)}$ \\
\hline & $\Pi^{(0)}=\{(0,2),(1,1)\}$ & $\left\{c_{0,1}=\frac{1}{2+\boldsymbol{i} \pi}\right\}$ \\
\hline & $r=1, m_{1}=2, \beta_{1}=\frac{1}{2}$ & $\mathcal{P}_{1}(z):=z^{2}-\frac{4+2 \boldsymbol{i} \pi}{\pi\left(-6-5 \boldsymbol{i} \pi+\pi^{2}\right)}$ \\
\hline & $\Pi^{(1)}=\{(1,1),(3,0)\}$ & $\left\{c_{1, \ell}=-(-1)^{\ell} \frac{1+\boldsymbol{i}}{\sqrt{\pi(\pi-3 \boldsymbol{i})}}\right\}$ \\
\hline
\end{tabular}

TABLE 2

Results summary for the quasi-polynomial (11).

According to Proposition 3.9, the solutions of the quasi-polynomial (11) around the critical point $(\boldsymbol{i}, \pi)$, split into two branches:

$$
\begin{aligned}
& s_{0,1}(\tau)=\boldsymbol{i}+\frac{1}{2+\boldsymbol{i} \pi}(\tau-\pi)+o(\tau), \\
& s_{1, \ell}(\tau)=\boldsymbol{i}-(-1)^{\ell} \frac{1+i}{\sqrt{\pi(\pi-3 \boldsymbol{i})}}(\tau-\pi)^{1 / 2}+o\left(\tau^{1 / 2}\right), \quad \ell=1,2 .
\end{aligned}
$$

Finally, since for the solution $s^{*}=\boldsymbol{i}$ at $\tau^{*}=\pi$ we have that $\beta_{0}=1 / m_{0}$ and $\beta_{1}=1 / m_{1}$, according to Proposition 3.7, such a solution has the CRS property. Furthermore, since the solutions are given in two branches with multiplicities $m_{0}$ and $m_{1}$, thus by Propositions 3.11 and 3.12 , one root will behave as a Taylor series $\left(s_{0,1}\right.$ in Figure 3-(b)), whereas the other branch will behave as a Puiseux series $\left(s_{1,1}\right.$ and $s_{1,2}$ in Figure $\left.3-(b)\right)$. 
EXAMPLE 4.2. Consider the following quasi-polynomial (borrowed from [13]):

$$
f(s, \tau)=\left(s^{4}+2 s^{2}+2\right)+2 e^{-s \tau}+e^{-2 s \tau},
$$

with a critical point at $\left(s^{*}, \tau^{*}\right)=(\boldsymbol{i}, \pi)$, and multiplicity $m=2$. Now, in order to determine the constants $n_{i}$, we compute (9) and thus, one gets:

$$
\begin{aligned}
& \left.\frac{\partial f}{\partial \tau}\right|_{(\boldsymbol{i}, \pi)}=0,\left.\quad \frac{\partial^{2} f}{\partial \tau^{2}}\right|_{(\boldsymbol{i}, \pi)}=-2 \quad \Rightarrow n_{0}=2, \\
& \left.\frac{\partial f}{\partial s}\right|_{(\boldsymbol{i}, \pi)}=0,\left.\quad \frac{\partial^{2} f}{\partial \tau \partial s}\right|_{(\boldsymbol{i}, \pi)}=2 \boldsymbol{i} \pi \Rightarrow n_{1}=1,
\end{aligned}
$$

and since $n_{0}<\infty$, this implies that $\kappa=0$. Then, the points on the Newton polygon for the quasi-polynomial $f$ at the critical point $(\boldsymbol{i}, \pi)$ are given by the following set:

$$
\Pi=\left\{\left(0, n_{0}\right),\left(1, n_{1}\right),(2,0)\right\}=\{(0,2),(1,1),(2,0)\} .
$$

After applying the Algorithm 1, we obtain the results summarized in Table 3.

TABLE 3

Results summary for the quasi-polynomial (27).

\begin{tabular}{lll}
\hline Initial Data & Algorithm Output & $\mathcal{Z}:=\left\{z \in \mathbb{C}: \mathcal{P}_{j}(z)=0\right\}$ \\
\hline$m=2, n_{0}=2$ & $r=1, m_{0}=m=2 \beta_{0}=1$ & $\mathcal{P}_{0}(z):=z^{2}+\frac{4 i \pi}{2 \pi^{2}-8} z-\frac{2}{2 \pi^{2}-8}$ \\
$\Pi=\{(0,2),(1,1),(2,0)\}$ & $\Pi^{(0)}=\{(0,2),(1,1),(2,0)\}$ & $\left\{c_{0,1}=-\frac{i}{\pi+2}, c_{0,2}-\frac{i}{\pi-2}\right\}$ \\
\hline
\end{tabular}

Since $n_{0}>n_{1}$ we are able to use Proposition 3.3, to obtain the first approximation of the Weierstrass polynomial $W(s, \tau)=s^{2}+w_{1} s+w_{0}$. Thus, the coefficients $w_{i}(\tau)$ are given by

$$
w_{0}(\tau)=\frac{-2}{2 \pi^{2}-8} \tau^{2}+o\left(\tau^{2}\right), \quad w_{1}(\tau)=\frac{4 i \pi}{2 \pi^{2}-8} \tau+o(\tau) .
$$

Now, following Proposition 3.7 the solutions of the quasi-polynomial $f(s, \tau)(27)$ around the critical pair $\left(s^{*}, \tau^{*}\right)=(\boldsymbol{i}, \pi)$ have only one branch $(r=1)$. Moreover, since $\beta=1$ and the solutions of $\mathcal{P}_{0}, c_{0,1}$ and $c_{0,2}$ are simple roots $(\mu=1)$, Proposition 3.11 implies that the solutions can be expanded as the following Taylor series:

$$
s_{0,1}(\tau)=\boldsymbol{i}-\frac{\boldsymbol{i}}{\pi+2}(\tau-\pi)+o(\tau), \quad s_{0,2}(\tau)=\boldsymbol{i}-\frac{\boldsymbol{i}}{\pi-2}(\tau-\pi)+o(\tau) .
$$

Finally, we have that $m_{0}=2$ and $\beta_{0}=1>1 / m_{0}$ then the solution $s=\boldsymbol{i}$ posses the NRS property. The asymptotic behavior is illustrated in Figure 3-(a).

EXAMPLE 4.3. Consider the following quasi-polynomial:

$$
f(s, \tau)=\left(s^{4}+3 s^{2}+2\right)+\left(s^{2}+1\right) e^{-s \tau},
$$

with critical root at $(\boldsymbol{i}, \pi)$ and multiplicity $m=2$. For this example we have that:

$$
\frac{\partial^{n_{0}} f}{\partial \tau^{n_{0}}} \equiv 0, \forall n_{0} \in \mathbb{N} \Rightarrow n_{0}=\infty,\left.\quad \frac{\partial^{2} f}{\partial \tau \partial s}\right|_{(\boldsymbol{i}, \pi)}=-2 \Rightarrow n_{1}=1 .
$$




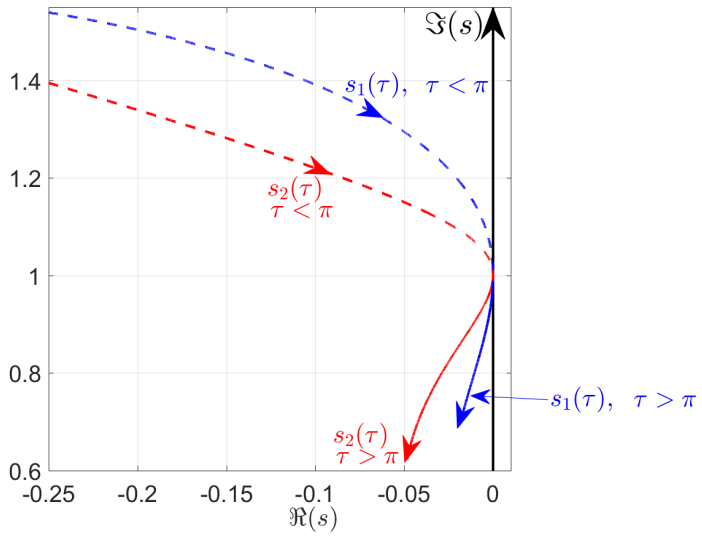

(a)

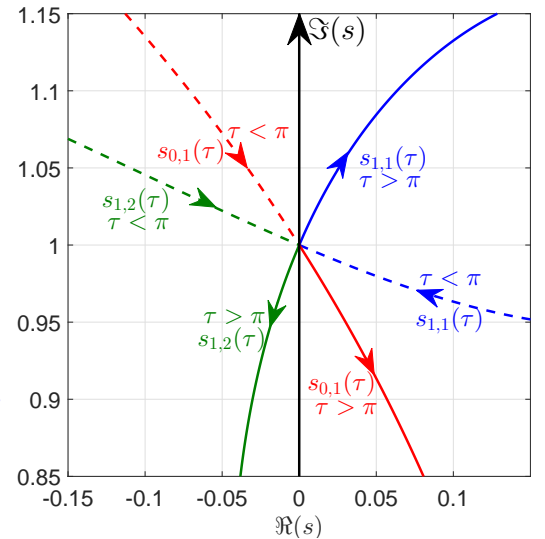

(b)

FiguRE 3. Root locus behavior: (a) for quasi-polynomial (27) and, (b) quasi-polynomial (11).

Since $n_{0}$ is not bounded and $n_{1}$ is finite, we have that $\kappa=1$, implying that we have 1 -invariant solution at $s=\boldsymbol{i}$ (denoted as $s_{\kappa, 1}$ in Figure 3a). Therefore, under these conditions the Weierstrass polynomial associated to $f(s, \tau)$ at $(\boldsymbol{i}, \tau)$ is given by

$$
W(s, \tau)=(s-\boldsymbol{i})\left((s-\boldsymbol{i})+w_{1}(\tau)\right) .
$$

For the remaining solution, we apply Algorithm 1. Table 4 summarizes the results.

TABLE 4

Results summary for the quasi-polynomial (28).

\begin{tabular}{lll}
\hline Initial Data & Algorithm Output & $\mathcal{Z}:=\left\{z \in \mathbb{C}: \mathcal{P}_{j}(z)=0\right\}$ \\
\hline$m=2, n_{1}=1$ & $r=1, m_{0}=1, \beta_{0}=1$ & $\mathcal{P}_{0}(z):=z-\frac{4}{-8+4 \boldsymbol{i} \pi}$ \\
$\Pi=\{(1,1),(2,0)\}$ & $\Pi^{(0)}=\{(1,1),(2,0)\}$ & $\left\{c_{0,1}=\frac{1}{-2+\boldsymbol{i} \pi}\right\}$ \\
\hline
\end{tabular}

Next, in the light of Proposition 3.9, we have:

$$
s_{1}(\tau)=\boldsymbol{i}-\frac{2+\boldsymbol{i} \pi}{4+\pi^{2}}(\tau-\pi)+o(\tau)
$$

In addition, since $\beta=1$ and $c_{0}$ is a simple root of $\mathcal{P}_{0}$, by a direct application of Proposition 3.11 we conclude that $s_{1}$ can be expanded as a Taylor series. Such a behavior is illustrated in Figure $3 \mathrm{a}$.

EXAMPLE 4.4. Consider the quasi-polynomial,

$$
f(s, \tau)=\left(s^{6}+3 s^{4}+3 s^{2}+2\right)+2 e^{-s \tau}+e^{-2 s \tau},
$$

where $s=\boldsymbol{i}$ is a double root at $\tau=\pi$. As in the previous examples, we have:

$$
\left.\frac{\partial^{2} f}{\partial \tau^{2}}\right|_{(\boldsymbol{i}, \pi)}=-2 \Rightarrow n_{0}=2,\left.\quad \frac{\partial^{2} f}{\partial \tau \partial s}\right|_{(\boldsymbol{i}, \pi)}=2 \boldsymbol{i} \pi \Rightarrow n_{1}=1
$$

After applying Algorithm 1 along with Proposition 3.9, Table 5 summarizes the results: 


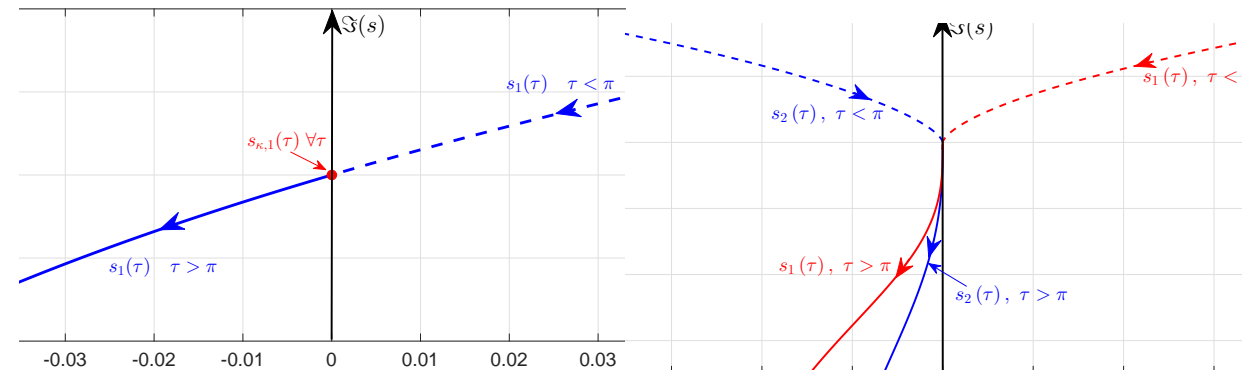

(a)

FIGURE 4. Root locus behavior: (a) for quasi-polynomial (28) and, (b) quasi-polynomial (29).

TABLE 5

Results summary for the quasi-polynomial (29).

\begin{tabular}{lll}
\hline Initial Data & Algorithm Output & $\mathcal{Z}:=\{z \in \mathbb{C}: \mathcal{P}(z)=0\}$ \\
\hline$m=2, n_{0}=2$ & $r=1, m_{0}=2, \beta_{0}=1, i_{-1}=0$ & $\mathcal{P}_{0}(z):=z^{2}+\frac{2 \boldsymbol{i}}{\pi} z-\frac{1}{\pi^{2}}$ \\
$\Pi=\{(0,2),(1,1),(2,0)\}$ & $\Pi^{(0)}=\{(0,2),(1,1),(2,0)\}$ & $\left\{c_{0,1}=\frac{-\boldsymbol{i}}{\pi}\right\}$ \\
\hline
\end{tabular}

Observe that since $\Re\left(c_{0,1}\right) \equiv 0$ we do not have enough information to determine the crossing directions. Now, from Table 5 , it can be seen that $\beta_{0}=1$ and $c_{0,1}$ is a multiple solution of $\mathcal{P}_{0}$ with multiplicity $m_{0}=2$. Hence, in the light of Proposition 3.13, we compute $\frac{d^{\nu_{j}+1}}{\tau^{\nu_{j}+1}} f\left(c_{j \sigma} \tau, \tau\right)$. Taking into account that $\nu_{0}=n_{i_{-1}}+i_{-1}=2$, we have:

$$
\left.\frac{d^{3}}{d \tau^{3}} f\left(-\frac{\boldsymbol{i} \tau}{\pi}, \tau\right)\right|_{\tau=0}=\frac{48}{\pi^{3}}
$$

Since we fulfill all hypothesis of Proposition 3.13, we are able to compute the higherorder terms. For this purpose, let us consider the function $f_{1}$ (see Remark 3.14);

$$
f_{1}\left(s_{1}, \tau\right):=\frac{1}{\tau^{2}} f\left(\tau\left(s_{1}-\frac{i}{\pi}\right), \tau\right)
$$

where we assumed that the critical point $(\boldsymbol{i}, \pi)$ has been shifted to the origin. Thus, a direct application of Algorithm 1 to $f_{1}$ gives the results summarized in Table 6.

TABLE 6

Results summary for the quasi-polynomial (30).

\begin{tabular}{lll}
\hline Initial Data & Algorithm Output & $\mathcal{Z}:=\{z \in \mathbb{C}: \mathcal{P}(z)=0\}$ \\
\hline$m=2, n_{0}=1$ & $r=1, m_{0}=2, \beta_{0}^{(1)}=1 / 2$ & $\mathcal{P}_{0}(z):=z^{2}+\frac{8}{\pi^{5}}$ \\
$\Pi=\{(0,1),(1,1),(2,0)\}$ & $\Pi^{(0)}=\{(0,1),(2,0)\}$ & $\left\{c_{0, \sigma}=-(-1)^{\sigma} \boldsymbol{i}\left(\frac{8}{\pi^{5}}\right)^{1 / 2}\right\}$ \\
\hline
\end{tabular}

According to Proposition 3.13, the solutions of (11) can be expanded as:

$$
s_{\sigma}(\tau)=\boldsymbol{i}-\frac{\boldsymbol{i}}{\pi}(\tau-\pi)+(-1)^{\sigma} \boldsymbol{i} \sqrt{\frac{8}{\pi^{5}}}(\tau-\pi)^{1+1 / 2}+o\left(\tau^{2}\right), \quad \sigma=1,2
$$


Finally, since $\beta_{0}=1>1 / m_{0}$ the solution $s=i$ has the NRS property, this behavior is illustrated in Figure $4 \mathrm{~b}$.

EXAMPLE 4.5. Our final example considers the quasi-polynomial,

$$
f(s, \tau)=p_{0}(s)+p_{1}(s) e^{-\tau s}-195.5224416 e^{-2 s \tau},
$$

where

$$
\begin{aligned}
& p_{0}(s):=7.5132152 s^{4}-15.793592 s^{3}+387.1503697 s^{2}-398.192295 s+4273.394627, \\
& p_{1}(s):=13.11465971 s^{2}-13.78421751 s-305.3204884 .
\end{aligned}
$$

In this case, for $\tau^{*}=1.902851533$, $f$ has two critical roots at $\pm \boldsymbol{i} \omega_{1}= \pm \boldsymbol{i} 4.059829001$ and $\pm \boldsymbol{i} \omega_{2}= \pm \boldsymbol{i} 5.73041116$, both of multiplicity $m=2$. Following similar steps as in the previous examples, we have for both critical points:

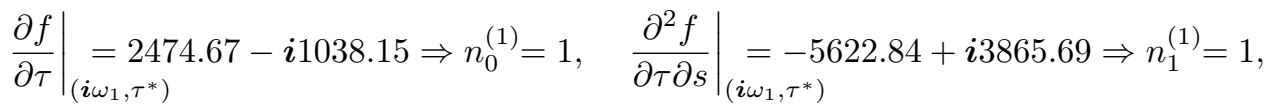

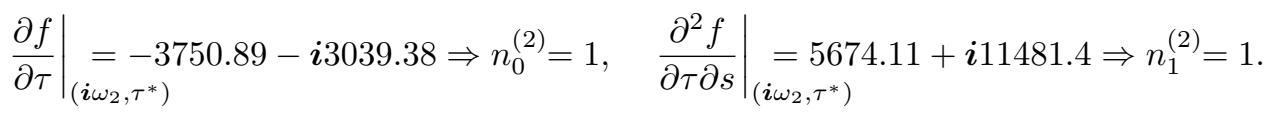

In the above computations we have adapted the notations $n_{i}^{(\ell)}$, where the super index $\ell$ relates the critical root $i \omega_{\ell}$, for $\ell \in\{1,2\}$. After applying Algorithm 1 along with

\begin{tabular}{|c|c|c|}
\hline Initial Data & Algorithm Output & $\mathcal{Z}:=\left\{z \in \mathbb{C}: \mathcal{P}_{j}(z)=0\right\}$ \\
\hline $\begin{array}{l}m=2, n_{0}^{(1)}=n_{0}^{(2)}=1 \\
\Pi=\{(0,1),(1,1),(2,0)\}\end{array}$ & $\begin{array}{l}r=1, m_{0}=m=2, \beta_{0}=\frac{1}{2} \\
\Pi^{(0)}=\{(0,1),(2,0)\}\end{array}$ & $\begin{array}{l}\mathcal{P}_{0}(z):=z^{2}+(0.290279-i 2.24248) \\
\left\{c_{0, \sigma}=(-1)^{\sigma}(0.9927+i 1.12948)\right\}\end{array}$ \\
\hline$\Pi=\{(0,1),(1,1),(2,0)\}$ & $\begin{array}{l}r=1, m_{0}=m=2, \beta_{0}=\frac{1}{2} \\
\Pi^{(0)}=\{(0,1),(2,0)\}\end{array}$ & $\begin{array}{l}\mathcal{P}_{0}(z):=z^{2}+(0.290279-\boldsymbol{i} 3.16524) \\
\left\{c_{0, \sigma}=(-1)^{\sigma}(1.20172+\boldsymbol{i} 1.31697)\right\}\end{array}$ \\
\hline
\end{tabular}
Proposition 3.9, Table 7 summarizes the results for both roots:

TABLE 7

Results summary for the quasi-polynomial (31).

According to Proposition 3.13, both solutions of (31) can be expanded as:

$$
\begin{aligned}
& s_{1, \sigma}(\tau)=\boldsymbol{i} 4.0598+(-1)^{\sigma}(0.9927+i 1.1294)(\tau-1.9028)^{1 / 2}+o\left(|\tau-1.9028|^{1 / 2}\right), \\
& s_{2, \sigma}(\tau)=\boldsymbol{i} 5.7304+(-1)^{\sigma}(1.2017+i 1.3169)(\tau-1.9028)^{1 / 2}+o\left(|\tau-1.9028|^{1 / 2}\right),
\end{aligned}
$$

where $\sigma \in\{1,2\}$. Finally, since in both cases $\beta_{0}=1 / 2$ and $m_{0}=m$, we conclude that both solutions have the CRS property. Moreover, since the solutions have the CRS property, according to Corollary 3.8 (i), we know that one of the solutions (for each $\left.\boldsymbol{i} \omega_{\ell}\right)$ will enter the RHP, whereas the other one will enter the LHP. Now, it is worth mentioning that the periodicity of $e^{i \omega}$ implies that $i \omega_{\ell}$ will be also a critical root for all $\tau \in\left\{\tau^{*}+\frac{2 k \pi}{\omega_{\ell}}: k \in \mathbb{N}\right\}$. However, in such a case the critical roots will be simple. We have shown several solutions $s_{\ell, k}(\tau), k \in \mathbb{N}, \ell \in\{1,2\}$ all of them simples, for $\tau \in(2.7,5.1)$ and each $s_{\ell, k}$ touches the imaginary axis exactly at the critical delay values $\tau(\ell, k)=\tau^{*}+\frac{2 k \pi}{\omega_{\ell}}$. 
5. Concluding Remarks. This paper is devoted to the analysis of the asymptotic behavior of multiple imaginary roots for quasi-polynomials of retarded-type. The presented approach is based on the Weierstrass Preparation Theorem which allows to deeply analyze the local behavior of a given critical solution. Some algebraic properties have been presented to characterize the branch structure for all critical solutions of the quasi-polynomial $f(s, \tau)$. In addition, we have shown that the leading terms of the Puiseux (or Taylor) expansions can be derived in a simple manner by computing the solutions of a given polynomial $\mathcal{P}_{j}$. We have also presented a characterization for the critical solutions (CRS, RS, NRS). Such classification have been shown to be extremely useful in analyzing the stability behavior of such a solutions. Finally, we gave some insights concerning the higher-order terms for the Puiseux (or Taylor) expansion of the critical solutions. Such a characterizations have been shown to be very convenient to determine the series expansion nature.

Acknowledgements. The authors are grateful to the reviewers for their useful comments which have helped to improve the exposition of this paper. The research of J. Chen was supported in part by the Hong Kong RGC under Projects CityU 111613 and CityU 11260016; the work of C.-F. Méndez-Barrios has been supported by Centrale Supélec and COPOCyT, Mexico; the work of A. Martínez-González was financially supported by CONACyT, Mexico.

\section{REFERENCES}

[1] C Abdallah, Peter Dorato, J Benites-Read, and R Byrne. Delayed positive feedback can stabilize oscillatory systems. In American Control Conference, pages 3106-3107. IEEE, 1993.

[2] Tiaoyang Cai, Huaguang Zhang, Binrui Wang, and Feisheng Yang. The asymptotic analysis of multiple imaginary characteristic roots for lti delayed systems based on Puiseux-Newton diagram. International Journal of Systems Science, 45(5):1145-1155, 2014.

[3] Eduardo Casas-Alvero. Singularities of plane curves. Cambridge University Press, New York, 2000.

[4] Jie Chen, Peilin Fu, Silviu-Iulian Niculescu, and Zhihong Guan. An eigenvalue perturbation approach to stability analysis, part I: eigenvalue series of matrix operators. SIAM Journal on Control and Optimization, 48(8):5564-5582, 2010.

[5] Jie Chen, Peilin Fu, Silviu-Iulian Niculescu, and Zhihong Guan. An eigenvalue perturbation approach to stability analysis, part II: when will zeros of time-delay systems cross imaginary axis? SIAM Journal on Control and Optimization, 48(8):5583-5605, 2010.

[6] Jie Chen, Guoxiang Gu, and Carl N Nett. A new method for computing delay margins for stability of linear delay systems. Systems $\&$ Control Letters, 26(2):107-117, 1995.

[7] K. Engelborghs, T. Luzyanina, and D. Roose. Numerical bifurcation analysis of delay differential equations using DDE-BIFTOOL. ACM Transactions on Mathematical Software, 28:1-21, 2002.

[8] K. Engelborghs, T. Luzyanina, and G. Samaey. DDE-BIFTOOL v. 2.00: a Matlab package for bifurcation analysis of delay differential equations. Technical Report TW-330, Department of Computer Science, K.U.Leuven, Belgium, 2001.

[9] Keqin Gu, Jie Chen, and Vladimir L Kharitonov. Stability of time-delay systems. Springer Science \& Business Media, New York, 2003.

[10] J. K. Hale and S. M. Verduyn Lunel. Introduction to Functional Differential Equations. Springer, New York, 1993.

[11] Lars Hormander. An introduction to complex analysis in several variables. North-Holland Co., Amsterdam, 1973.

[12] R Hryniv and P Lancaster. On the perturbation of analytic matrix functions. Integral Equations and Operator Theory, 34(3):325-338, 1999.

[13] Elias Jarlebring and Wim Michiels. Invariance properties in the root sensitivity of time-delay systems with double imaginary roots. Automatica, 46(6):1112-1115, 2010.

[14] H Langer, B Najman, and K Veselić. Perturbation of the eigenvalues of quadratic matrix polynomials. SIAM Journal on Matrix Analysis and Applications, 13(2):474-489, 1992.

[15] X. G. Li, S. I. Niculescu, A. Çela, L. Zhang, and X. Li. A frequency-sweeping framework 
for stability analysis of time-delay systems. IEEE Transactions on Automatic Control, 62(8):3701-3716, 2017.

[16] Xu-Guang Li, Silviu-Iulian Niculescu, and Arben Cela. Analytic Curve Frequency-Sweeping Stability Tests for Systems with Commensurate Delays. SpringerBriefs in Control, Automation and Robotics. Springer International Publishing, 2015.

[17] Xu-Guang Li, Silviu-Iulian Niculescu, Arben Çela, Hong-Hai Wang, and Tiao-Yang Cai. On computing Puiseux series for multiple imaginary characteristic roots of lti systems with commensurate delays. IEEE Trans. Automat. Contr., 58(5):1338-1343, 2013.

[18] Alexei Abaevich Mailybaev and Samvel Samvelovich Grigoryan. On the Weierstrass preparation theorem. Matematicheskie Zametki, 69(2):194-199, 2001.

[19] C.F. Méndez-Barrios, S.-I. Niculescu, J. Chen, and V.M. Cárdenas-Galindo. On the Weierstrass preparation theorem with applications to the asymptotic analysis of characteristics roots of time-delay systems. In IFAC Workshop on Time Delay Systems, pages 251-256, Ann Arbor, 2015.

[20] Wim Michiels and Silviu-Iulian Niculescu. Stability, Control, and Computation for Time-Delay Systems. An Eigenvalue-Based Approach (2. ed.). Advances in design and control. SIAM, Philadelphia, 2014.

[21] B. V. Shabat. Introduction to Complex Analysis. Part II. Functions of Several Variables. Translations of Mathematical Monographs. American Mathematical Society, Providence, 1992.

[22] M. Vainberg and V. Trenogin. Theory of branching of solutions of non-linear equations. Noordhoff International Pub., Leyden, 1974

[23] Robert John Walker. Algebraic Curves. Springer-Verlag, New York, 1978.

[24] Charles Terence Clegg Wall. Singular points of plane curves. Cambridge University Press, New York, 2004. 